Conclusion: Renal transplantation is a safe alternative therapy for ESRD in this population and can provide a long-term survival. However, it is very important to consider the occurrence of flares even in the long-term post-transplant.

Figures 1 and 2.

Disclosure of Interests: Lara Sanchez-Bilbao Grant/research support from: Pfizer, Marina de Cos-Gómez: None declared, Juan Carlos Ruiz-San Millán: None declared, Miguel A González-Gay Grant/research support from: Pfizer, Abbvie, MSD, Speakers bureau: Pfizer, Abbvie, MSD, Ricardo Blanco Grant/ research support from: AbbVie, MSD, and Roche, Speakers bureau: AbbVie, Pfizer, Roche, Bristol-Myers, Janssen, and MSD

DOI: 10.1136/annrheumdis-2020-eular.4873

\section{AB0444 THROMBOTIC MICROANGIOPATHY AND SJÖGREN SYNDROME, AN UNUSUAL ASSOCIATION. PRESENTATION OF CLINICAL CASE AND SYSTEMATIC REVIEW OF THE LITERATURE}

Y. Santamaria ${ }^{1}$, M. Galvis ${ }^{1}$, A. Vanegas ${ }^{1} .{ }^{1}$ Universidad de Antioquia, Medellín, Colombia

Background: Thrombotic microangiopathy (TMA) is a clinicopathologic diagnosis defined as microangiopathic hemolytic anemia (MAHA) with associated features of thrombocytopenia and end-organ ischemia. Systemic lupus erythematosus, antiphospholipid antibody syndrome, and scleroderma, are within the autoimmune diseases, the more commonly associated with TMA. It has been considered that the association with Sjögren Syndrome (SS) is rare.

Objectives: To describe one patient with TMA and SS, and to review all cases reported in the literature.

Methods: We notified a clinical case of a patient with Sjögren's syndrome and TMA. Then, we searched the medical literature finding a total of 17 cases reported with this association until 2019. Before the data obtained were tabulated by trained staff and descriptive, comparing groups and bivariate analysis was performed. The outcome of interest was the death of the patient. Stata 12.0 software was used.

Results: A 26-years-old Colombian female presented with a 6-week history of petechiae in lower limbs, gingivorrhagia, menorrhagia and jaundice; and previous history of arthritis and xerostomia. On admission, in the context of severe thrombocytopenia and MAHA, MAT diagnosis was made. During hospitalization we confirm the diagnosis of SS with ANA, antiRo and salivary gland biopsy. The patient was treated with steroids (methylprednisolone $500 \mathrm{mg} /$ day, 3 days), plasma exchange therapy (PLEX) and Cyclophosphamide $(750 \mathrm{mg})$, with recovery of hemoglobin and platelet levels; however, the patient died due to a complication of the PLEX catheter removal procedure.

A total of 18 patients diagnosed with de novo or prevalent Sjögren's syndrome who had hospital admission with a diagnosis of TMA were included. The mean age was 54.55 years (Standard deviation (SD): 12.45 ) and $83.33 \%$ of the patients corresponded to the female gender.

At admission, the mean of hemoglobin was $8.45 \mathrm{~g} / \mathrm{dL}$ (SD: 2.55) and median platelets of $27250 / \mathrm{mm} 3$ (interquartile range (IQR) 10500 - 102000) were found. The most frequent clinical manifestations were central nervous system alterations $(50 \%)$, followed by bleeding in the skin and renal failure $(44.44 \%)$ and fever $(27.78 \%)$. The most frequent antibodies found were anti-Ro (100\%), anti-nuclear antibodies $(80 \%)$ and anti-La $(75 \%)$. The most frequently prescribed treatment was plasma exchange therapy $(83.33 \%)$, intravenous steroids $(61.11 \%)$, oral steroids $(61.11 \%$ ) and cyclophosphamide (27.78\%). Of the total patients, $38.89 \%$ died and $27.7 \%$ had some relapse of TMA.

In the group comparison analysis, differences were found in intravenous steroid (81.82\% in those who lived vs. $28.57 \%$ in those who died $p=0.039$ ), use of PLEX (100\% in those who survived vs. $57.14 \%$ in those who died $p=0.043$ ), fever $(9.09 \%$ in those who survived and $57.14 \%$ in those who died, $p=0.047)$, admission hemoglobin $(7.65 \mathrm{~g} / \mathrm{dL}$ in those who lived vs. $10.22 \mathrm{~g} / \mathrm{dL}$ in those who died, $p=0.05)$, final platelets $(148,000$ in which who lived and 39,000 in those who died $p=0.02$ ). Then, in the logistic regression analysis, an association was found between mortality and use of intravenous steroids (OR: 0.08, 95\% $\mathrm{Cl} 0.009$ $0.83, p=0.35$ ) and fever at admission (OR: $13.3395 \% \mathrm{Cl}: 1.04-169.55, \mathrm{p}=$ 0.046).

Conclusion: While the association between TMA and SS is uncommon, so far 18 cases have been reported in the world medical literature. It is typically a condition of women age close to 50 years. The most frequent manifestations are neurological. Among the variables evaluated, only the use of endovenous steroids was associated with a decrease in the probability of mortality; on the contrary, the presence of fever at hospital admission increased the probability of death. The results should be evaluated with caution, since, due to the limited availability of information, they may not be generalizable to clinical practice. More information on this should be obtained in the future.

Disclosure of Interests: None declared

DOI: 10.1136/annrheumdis-2020-eular.6576

\section{$\mathrm{AB} 0446$ \\ CLINICAL AND IMMUNOLOGICAL CHARACTERISTICS OF 484 SYSTEMIC LUPUS ERYTHEMATOSUS PATIENTS IN XINJIANG OF CHINA: A COMPARATIVE ANALYSIS}

Y. M. Shi' ${ }^{1}$ X. Wu ${ }^{1}$, L. Wu ${ }^{1}$, C. N. Luo ${ }^{1} .{ }^{1}$ People's Hospital of the Xinjiang Uygur Autonomous Region, Urumqi, China

Background: Systemic lupus erythematosus (SLE) is a chronic inflammatory autoimmune disease. Epidemiological studies in SLE have been reported in the literature in many countries and ethnic groups. Although SLE in China has been described in the past, there has not been a detailed evaluation of SLE patients in Xinjiang of China, a largely Uygur population.

Objectives: To describe the clinical featuresand immunological features of 484 SLE subjects.

Methods: 484 adult patients followed in the The People's Hospital of the Xinjiang Uygur Autonomous Region, 211 patients with Uygur SLE as well as 273patients with Han SLE.

Results: Of the 211 Uygur SLE patients, 195 (92.4\%) were female and 16 $(7.6 \%)$ were male (female:male $=12.2: 1$ ), the mean age at SLE onset was $34.67 \pm 11.57$ years, mean disease duration was $20.77 \pm 35.16$ months. Of the 273 Han SLE patients, $247(90.5 \%)$ were female and $26(9.5 \%)$ were male, the mean age at SLE onset was $36.68 \pm 14.44$ years, mean disease duration was $41.64 \pm 61.89$ months.(2)between the Uygur and Han SLE patients, the Raynaud phenomenon $\left(x^{2}=6.066 P=0.014\right)$, Chest pain $\left(x^{2}=7.906 P=0.005\right)$, headache $\left(x^{2}=4.572 \quad P=0.029\right)$ has obvious differences (table 1).(3)The anti-nuclear $\left(x^{2}=8.108 \mathrm{P}=0.004\right)$, anti-AHA $\left(x^{2}=4.952 \mathrm{P}=0.026\right)$ were higer in Uygur SLE patients than those in Han SLE patients; the Uygur SLE patients has been anemia $\left(x^{2}=6.904, P=0.009\right)$, high level of immunoglobulin $\left(X^{2}=8.939, P=0.003\right)$, decrease of complement $\left(X^{2}=6.330 \quad P=0.012\right)$. (table 2)

figure 1. Clinical manifestations

\begin{tabular}{lcccc}
\hline Clinical manifestations & Uygur SLE patients $(\mathrm{n}=211)$ & Han SLE $(\mathrm{n}=273)$ & $x^{2}$ & $P$ \\
\hline rash & $106(50.2 \%)$ & $157(57.5 \%)$ & 2.537 & 0.111 \\
Photosensitivity & $4(19 \%)$ & $56(20.5 \%)$ & 0.181 & 0.670 \\
Alopecia & $73(34.6 \%)$ & $101(37.0 \%)$ & 0.298 & 0.585 \\
Oral ulcers & $49(23.2 \%)$ & $64(23.5 \%)$ & 0.006 & 0.937 \\
Raynaud phenomenon & $28(13.3 \%)$ & $60(22 \%)$ & 6.066 & 0.014 \\
livedo reticularis & $5(2.4 \%)$ & $7(2.6 \%)$ & 0.020 & 0.866 \\
arthralgia/arthritis & $105(49.8 \%)$ & $159(58.5 \%)$ & 3.623 & 0.057 \\
abnormal liver-function & $8(3.8 \%)$ & $7(2.6 \%)$ & 0.597 & 0.440 \\
Chest pain & $28(13.3 \%)$ & $16(5.9 \%)$ & 7.906 & 0.005 \\
Suffocation & $49(23.2 \%)$ & $52(19 \%)$ & 1.256 & 0.262 \\
palpitation & $27(12.8 \%)$ & $41(15 \%)$ & 0.487 & 0.485 \\
Shortness of breath & $24(11.4 \%)$ & $35(12.8 \%)$ & 0.233 & 0.630 \\
Ophthalmia & $2(0.9 \%)$ & $6(2.2 \%)$ & 1.154 & 0.283 \\
Visual impairment & $1(0.5 \%)$ & $3(1.1 \%)$ & 0.567 & 0.457 \\
hemiplegia & $1(0.5 \%)$ & $0(0.0 \%)$ & 1.297 & 0.436 \\
Mental disorder & $4(1.9 \%)$ & $11(4.0 \%)$ & 1.804 & 0.179 \\
headache & $14(6.6 \%)$ & $7(2.6 \%)$ & 4.572 & 0.029 \\
Lower Limb Edem & $36(17.1 \%)$ & $34(12.5 \%)$ & 2.042 & 0.153 \\
pleurisy & $37(17.5 \%)$ & $43(15.8 \%)$ & 0.256 & 0.613 \\
pericarditis & $38(18 \%)$ & $33(12.1 \%)$ & 3.273 & 0.070 \\
pulmonary fibrosis & $7(3.3 \%)$ & $9(3.3 \%)$ & 0.000 & 0.982 \\
\hline
\end{tabular}

figure 2. immunological manifestations

\begin{tabular}{lcccc}
\hline manifestations & Uygur SLE patients(n=211) & Han SLE $(\mathrm{n}=273)$ & $x^{2}$ & $P$ \\
\hline antinuclear antibodies & $185(87.7 \%)$ & $212(77.7 \%)$ & 8.108 & 0.004 \\
anti-dsDNA & $115(54.5 \%)$ & $144(52.7 \%)$ & 0.147 & 0.701 \\
anti-SSA & $101(47.9 \%)$ & $149(54.6 \%)$ & 2.147 & 0.143 \\
anti-SSB & $45(21.3 \%)$ & $63(23.1 \%)$ & 0.210 & 0.647 \\
anti-Sm & $44(21 \%)$ & $68(25 \%)$ & 1.088 & 0.297 \\
ACL & $29(13.8 \%)$ & $26(9.5 \%)$ & 2.161 & 0.142 \\
antiU1-RNP & $74(35.1 \%)$ & $100(36.6 \%)$ & 0.126 & 0.723 \\
anti-AHA & $60(28.4 \%)$ & $54(19.8 \%)$ & 4.952 & 0.026 \\
Low white blood cell & $46(22.1 \%)$ & $79(29 \%)$ & 2.938 & 0.087 \\
anemia & $90(42.9 \%)$ & $85(36.3 \%)$ & 6.904 & 0.009 \\
Thrombocytopenia & $36(17.1 \%)$ & $51(18.7 \%)$ & 0.212 & 0.645 \\
Urine protein positive & $84(39.8 \%)$ & $114(41.9 \%)$ & 0.217 & 0.641 \\
Rise of urine RBC & $29(13.7 \%)$ & $35(12.8 \%)$ & 0.088 & 0.766 \\
Increased immunoglobulin & $82(38.9 \%)$ & $71(26.1 \%)$ & 8.939 & 0.003 \\
Complement decline & $120(57.1 \%)$ & $124(45.6 \%)$ & 6.330 & 0.012 \\
\hline
\end{tabular}

Conclusion: The Uygur SLE patients have their own clinical and immunological characteristics, which has guiding significance in the diagnosis, treatment and prognosis of SLE. 